3

4

5

6

8

\title{
Disseminating antibiotic resistance during treatment
}

\section{A multidrug efflux pump can help bacteria acquire antibiotic resistance through plasmid transfer}

\author{
By Vanessa R. Povolo ${ }^{1,2}$ and Martin Ackermann ${ }^{1,2}$ \\ ${ }^{1}$ Department of Environmental Systems Science, ETH Zurich, 8092 Zurich, Switzerland. \\ 2 Department of Environmental Microbiology, Eawag, 8600 Dübendorf, Switzerland. \\ Email: vanessa.povolo@eawag.ch, martin.ackermann@env.ethz.ch
}

The spread of antibiotic resistance in bacterial pathogens has become a major public health problem. Resistance genes to clinically relevant antibiotics are often carried on plasmids (circular pieces of DNA) that can be transferred between different types of bacteria through the process of conjugation (1). For example, bacteria that are exposed to antibiotics can survive if they have already received a plasmid with an antibiotic resistance gene from another bacterium. However, how this process of horizontal transfer of resistance genes in the presence of antibiotics works is not entirely clear. Many antibiotics inhibit gene expression, which means that bacteria that have received a plasmid containing an antibiotic resistance gene are unable to express it. On page XXX of this issue, Nolivos et al. (2) describe a mechanism by which bacteria can express resistance genes even in the presence of antibiotics that block gene expression.

Nolivos et al. developed an experimental system to follow the transfer of plasmids that contain antibiotic resistance genes at the single-cell level and in real time. They followed the transfer of a plasmid carrying a resistance gene to the antibiotic tetracycline from a resistant donor bacterium to a sensitive recipient. Resistance to tetracycline is mediated through the TetA efflux pump that exports tetracycline molecules from the cytoplasm (2). The authors found that the TetA protein is rapidly produced after the plasmid enters a recipient cell. This is surprising because tetracycline inhibits translation and thus gene expression.

How can a resistance gene be expressed in the presence of a translational inhibitor? One possible explanation is that the recipient not only receives a plasmid from the donor but also receives the TetA resistance protein at the same time. The transferred TetA protein could then pump out enough tetracycline to allow translation and hence the production of more TetA from the plasmid that is received.

Cotransfer of cytoplasmic proteins with plasmids is a recurring idea in conjugation research (3). However, so far there is no clear support for the transfer of cytoplasmic proteins other than those required for plasmid DNA transfer between donor and recipient. 
Consistent with this idea, Nolivos et al. found no evidence for TetA being transferred from the donor to the recipient cell. This suggests that another molecular mechanism explains how bacteria can produce resistance proteins in the presence of translation-inhibiting antibiotics.

Nolivos et al. demonstrate that the mechanism involves the bacterial multidrug efflux pump comprised of the three proteins acridine resistance protein $A(A c r A), A c r B$, and tolerance to colicine (TolC). This AcrAB-TolC efflux pump confers natural resistance to bile salts in Escherichia coli and provides resistance to a wide range of chemical compounds present in subinhibitory concentrations $(4,5)$. The authors showed that deletion of the genes encoding the subunits of this pump does not impede plasmid conjugation but prevents synthesis of the encoded resistance protein. These findings indicate that the AcrAB-TolC pump keeps the intracellular tetracycline concentration low enough to allow synthesis of the plasmid-encoded resistance protein after plasmid transfer in the presence of tetracycline. The active efflux of antibiotics through the AcrAB-TolC multidrug efflux pump also allows acquisition of resistance in the presence of other antibiotics that block gene expression, such as chloramphenicol, erythromycin, and rifampicin. These findings further emphasize the importance of the AcrAB-TolC multidrug efflux pump by revealing a new role in the acquisition of antibiotic resistance.

The findings of Nolivos et al. could potentially be relevant for other types of resistance evolution. For example, spontaneous mutations that confer antibiotic resistance often take three to four bacterial generations until the resistance manifests phenotypically (6), and multidrug-efflux pumps could allow bacteria to persist for long enough and express their mutated proteins. Similarly, these pumps could allow the expression of resistance genes acquired from viruses or by the uptake of free DNA.

This study also suggests a possible extension of our attempts to control bacterial infections. Bacterial infections in humans are commonly treated with antibiotics. Such treatment often leads to the emergence of newly resistant bacterial strains through horizontal gene transfer in the digestive tract $(8,9)$. This can potentially lead to treatment failure as well as to the evolution of new resistant pathogens. The rise of these newly resistant strains is probably caused by two factors: direct selection by the antibiotic and an increase in horizontal gene transfer from the increased pool of resistant bacteria in the digestive tract (9). In addition to trying to inhibit growth and survival of bacteria through antibiotics, one could thus also consider trying to reduce the horizontal transfer of antibiotic resistance (10).

The findings of Nolivos et al. show that inhibition of multidrug efflux systems could be an important element of such a strategy. Inhibitors of bacterial efflux pumps are receiving a lot of attention because they often make bacteria more sensitive to antibiotics (11). This study shows that these inhibitors could have a second, indirect, effect: They could block the transfer of resistance between bacteria during treatment. As a consequence, they could limit the emergence of new resistant strains, which pose a threat both to an individual patient as well as to the population at large. 
84

85 1. P. M. Hawkey, A. M. Jones, J. Antimicrob. Chemother. 64 (Suppl 1), i3 (2009).

86 2. S. Nolivos, J. Caryon, A. Dedieu, A. Page, F. Delolome, C. Lesterlin, Science 364, XXX

87 (2019).

88 3. W. Saenger, P. Orth, C. Kisker, W. Hillen, W. Hinrichs, Angew. Chem. Int. Ed. 39, 2042 89 (2000).

90 4. C. E. Rees, B. M. Wilkins, Mol. Microbiol. 4, 1199 (1990).

91 5. D. G. Thanassi, L. W. Cheng, H. Nikaido, J. Bacteriol. 179, 2512 (1997).

92 6. X. Z. Li, P. Plésiat, H. Nikaido, Clin. Microbiol. Rev. 28, 337 (2015).

93 7. L. Sun, H.K. Alexander, B. Bogos, D.J. Kiviet, M. Ackermann, S. Bonhoeffer, PLOS Biol. $94 \quad 16$, e2004644 (2018).

95 8. J. R. Huddleston, Infect. Drug Resist. 7, 167 (2014).

96 9. C. Jernberg, S. Löfmark, C. Edlund, J. K. Jansson, Microbiology 156, 3216 (2010).

97 10. M. Diard, e. Bakkeren, J.K. Cornuault, K. Moor, A. Hausmann, M.E. Sellin, C. Loverdo, 98 A. Aertsen, M. Ackermann, D. De Paepe, E. Slack, W.-D. Hardt, Science 355, 1211 (2017).

11. A. Lamut et al., Med. Res. Rev. (2019). 10.1002/ med.21591 